\title{
EFFECTS OF LASER PROCESSING ON DOMAIN WALL MOTION IN GRAIN-ORIENTED SILICON STEEL
}

\author{
T. BARRADI \\ S.E.E.I. SUPELEC, Plateau du Moulon, 91192 Gif/Yvette cedex, France
}

The influence of laser irradiation spacing on the shape of the so-called "saw-tooth" domains is reported. Dynamic behavior under an external field, applied in the rolling direction, is investigated using a suitable imaging package developed for magneto-optical Kerr effect studies. Results relative to irradiated and non-irradiated commercial $\mathrm{Hi}-\mathrm{B}$ specimens are confronted and the incidence on core losses is discussed.

KEY WORDS Fe-Si, grain oriented, laser irradiation, saw-tooth domains, magneto-optical Kerr effect.

\section{INTRODUCTION}

Domain refining is one of the most effective techniques to improve iron losses of grain-oriented $\mathrm{Si}-\mathrm{Fe}$ sheets. There are many investigations ${ }^{1-5}$ devoted to study domain patterns resulting from laser treatment. But the quantitative analysis of wall domain motion, which is necessary to estimate iron losses, was omitted.

The aim of this paper is to report some characteristics of static and dynamic behavior of some magnetic domains observed in locally annealed specimens, depending on the regularity of laser spot matrix and spot spacing. Furthermore, the collapse process of these typical domains is illustrated over a complete topological hysteresis loop. During the magnetization process, each domain wall motion is computed pixel by pixel and a histogram per wall, showing each individual pixel displacement, is displayed. Then, the average wall displacement, in one or several grains or even in the entire specimen, is deduced.

This analysis by the means of what we call "Kermag" package ${ }^{6}$ is accomplished on magneto-optical images which are detected by a CCD or a high speed camera. Both irradiated and non-irradiated specimens are investigated in order to confront the corresponding results and conclude as to the impact of laser treatment on magnetic domain behavior.

\section{LASER PROCESS SPECIFICATIONS AND DOMAIN REFINEMENT}

For the irradiation processing, the laser beam was focused on the sample by a suitable microscope objective and the sample was mounted on a microposition- 
ning base fitted with ultraprecise two-axis positionning which provides the desired displacement. Two kinds of laser spot matrices are possible: regular or irregular. This possibility allows to investigate the domain's shape dependence on the neighbouring laser spot spacing.

The refinement is satisfactory when the distance between spots is optimal both vertically and horizontally (Figure 1a, b). But even in this case, some "saw-tooth" domains may appear (Figure 1c). When the spacing is not optimized, the

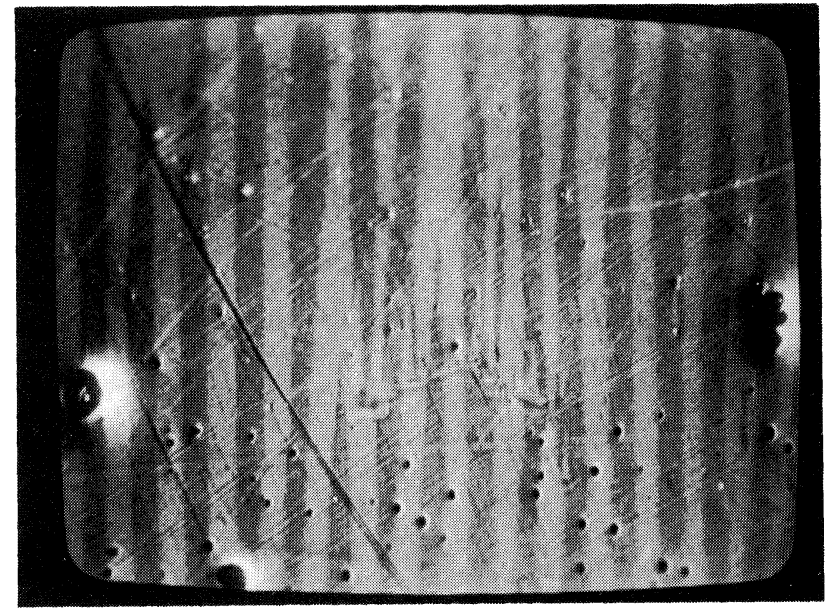

(a)

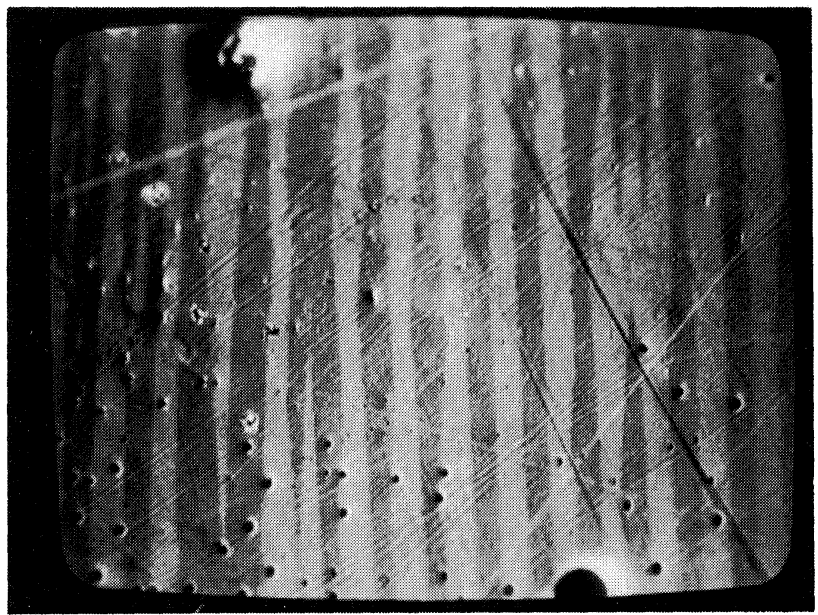

(b)

Figure 1a,b Regular refinement between optimally spaced laser spots. 


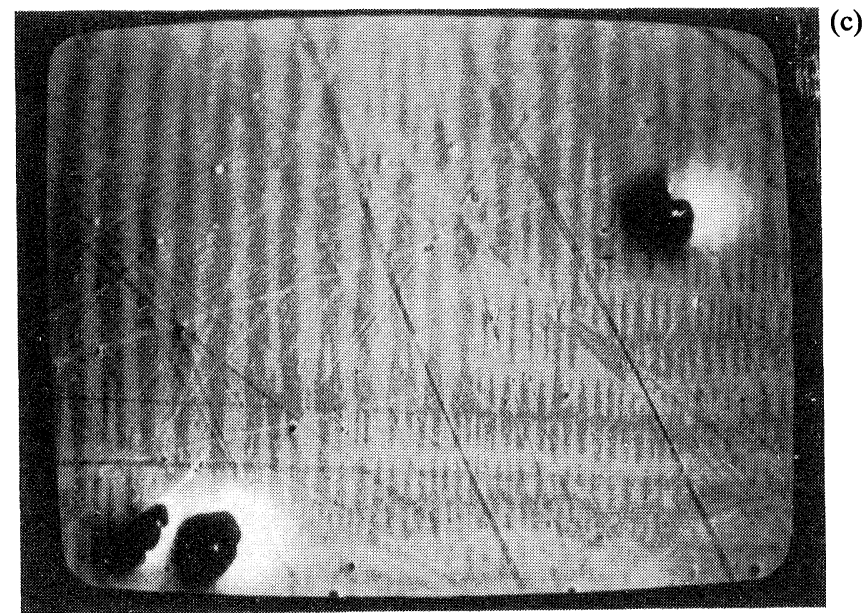

Figure 1c "Saw-tooth" domains between non-optimally spaced laser spots.

shape of domains is considerably distorted as illustrated by Figure 1d, where the saw-tooth character is more pronounced.

\section{DYNAMIC BEHAVIOR}

\section{III.1. General Considerations}

The field is applied in the rolling direction. The demagnetizing field is reduced to about $4 \%$ of the external field value by means of a special experimental set-up based on the single sheet tester principle.

The present investigation concerns, in non-irradiated sheet, both slab and

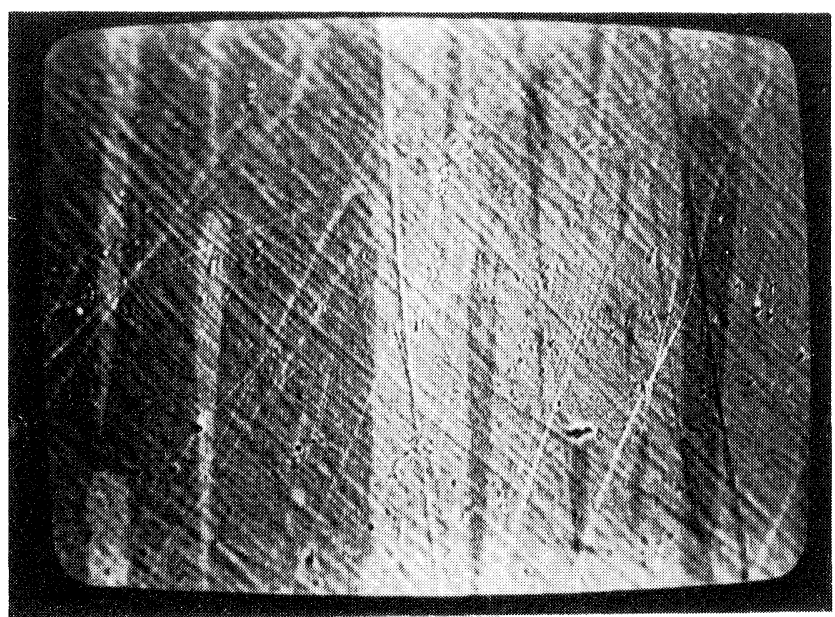

Figure 2 Domain pattern with supplementary structure (lancets). 


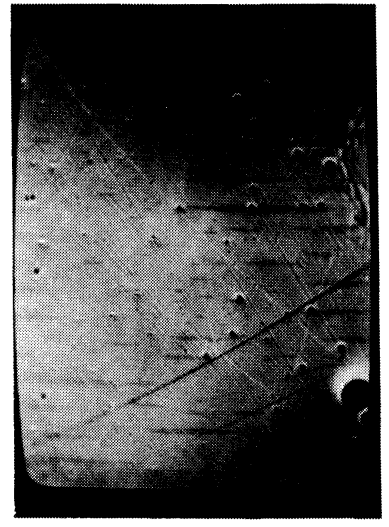

$$
=\frac{E}{k}
$$

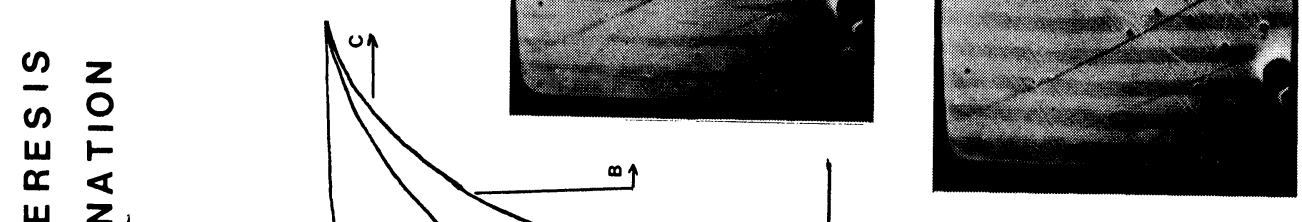

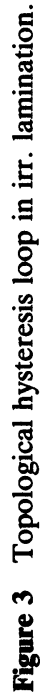

w z

\& $\Sigma$

I 4

$\begin{array}{ll}1 & 0 \\ 0 & \underline{1} \\ 0 & = \\ 0 & z \\ 0 & - \\ 1 & \\ 0 & 0 \\ 0 & 0 \\ 0 & 0 \\ 1 & 1\end{array}$
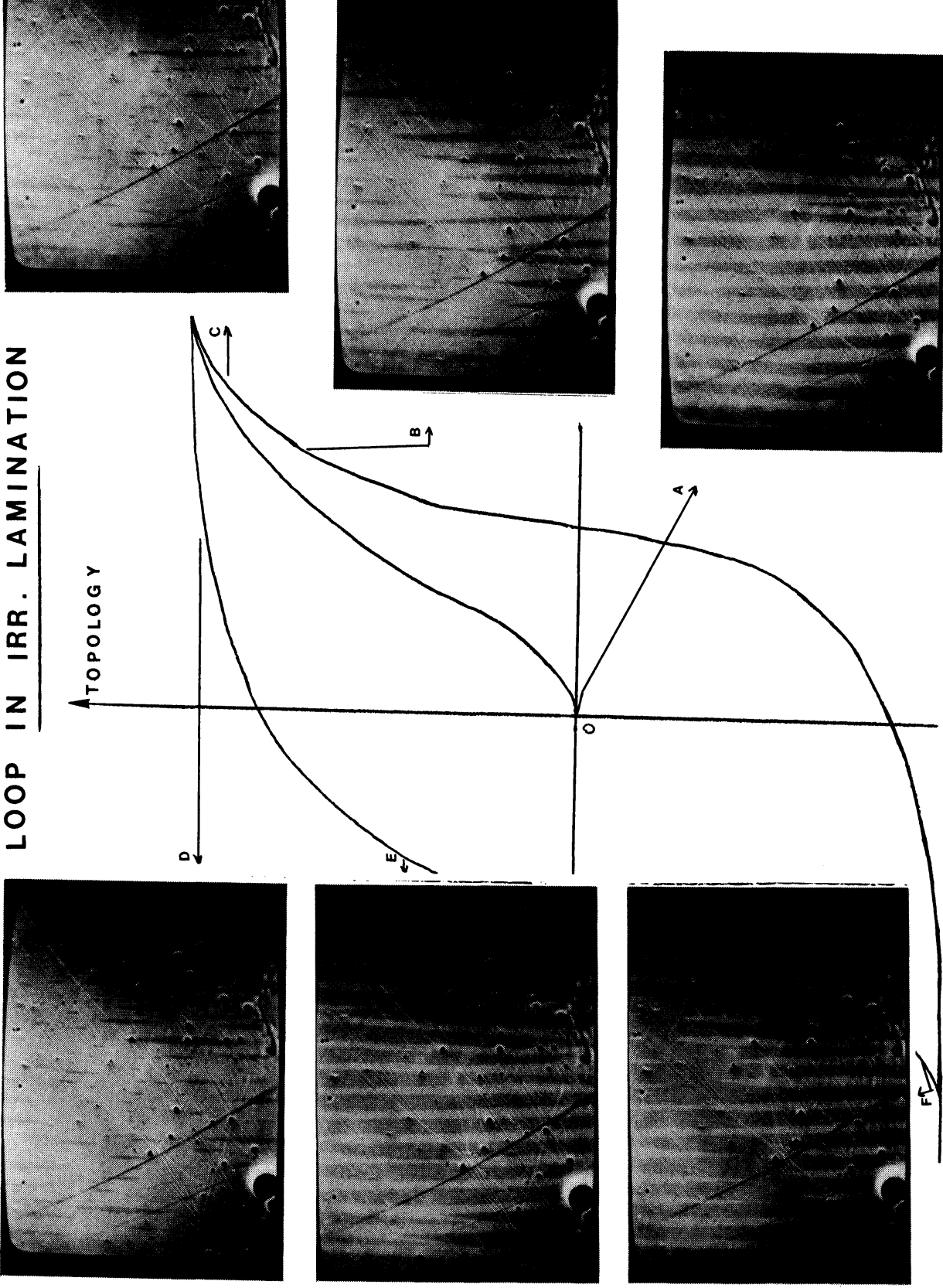
lancet domains which appear when the tilt angle of the [001] axis out of the crystal surface $(\beta)$ is greater than about $1.2^{\circ}$ (Figure 2 ).

After laser irradiation, the magnetic domains considered are like those shown by Figure $3 \mathrm{a}$ with or without supplementary structure depending on the misorientation value.

\section{III.2. Topological Evolution of Domains in Irradiated Sheet}

The initial domain configuration shown on picture $3 a$ corresponds to the completely demagnetized state in the $50 \mathrm{~Hz}$ applied field. Then, the desired field is applied but it was necessary to wait several periods, in order to eliminate transient phenomena, before taking the remaining pictures of the topological hysteresis loop illustrated by Figure 3 . Only the most significant states are reported. It is observed that, near saturation (Figure $3 b, 3 c, 3 f$ ), the domains collapse to a shape resembling an elongated losange. Likewise, the reverse domains are nucleated with the similar geometrical form (Figure 3d).

These observations are in agreement with the remarks appearing in a recent paper $^{5}$ which surmise that local stresses introduced by laser irradiation are possible centers for domain refining in grain oriented silicon steel.

This particular behavior, induced by the effect of irradiation, led us to undertake a fine study of wall motion before and after laser processing. As indicated previously, the practical means used is the "Kermag" package and, especially, the options which allow image subtraction and measurement of the pixel coordinates such as spatial position (accuracy $<0.4 \%$ ), grey level (accuracy $<0.2 \%$ ) and also pixel displacement.

\section{III.3. Experimental Characterization of Domain Wall Motion}

At each magnetization transition, the picture immediately preceding is chosen as the reference image (denoted Im 1); the following image (denoted Im 2) corresponds to the applied field with the desired waveform, value and direction. Consequently, the magnetic structure of domains evolves from one magnetization state to another such as the one illustrated by Figure $4 \mathrm{~b}$, for which the preceding state is shown by Figure 4a.

In order to compute quantitatively every change occurring at each state transition, the applied algorithm allows the computation of the various nuances in the grey level of those pixels having the same spatial coordinates in the previous images.

Thus, if $g_{i}$ is the grey level for the $i$ rank pixel $M_{i}$ of $\operatorname{Im} 1$, and $g_{i}^{\prime}$ the grey level for the same pixel in $\operatorname{Im} 2$, the grey level $g$ of the pixel having the same coordinates in $\operatorname{Im} 3$ as the previous ones, is simply the difference between $g_{i}$ and $\boldsymbol{g}_{i}^{\prime}$.

However, to avoid the appearance of unreal objects on the result image, which happens, for instance, when $g_{i}$ is less than $g_{i}^{\prime}$, a suitable formula is used to compute these singular points. Moreover, as shown by this algorithm, all objects common to Im 1 and Im 2 must disappear. It follows from this that, surface defects and optical aberrations are removed on the result picture. This fact is illustrated by the series of Figure 4 where $\operatorname{Im} 1$ and $\operatorname{Im} 2$ are voluntarily chosen 


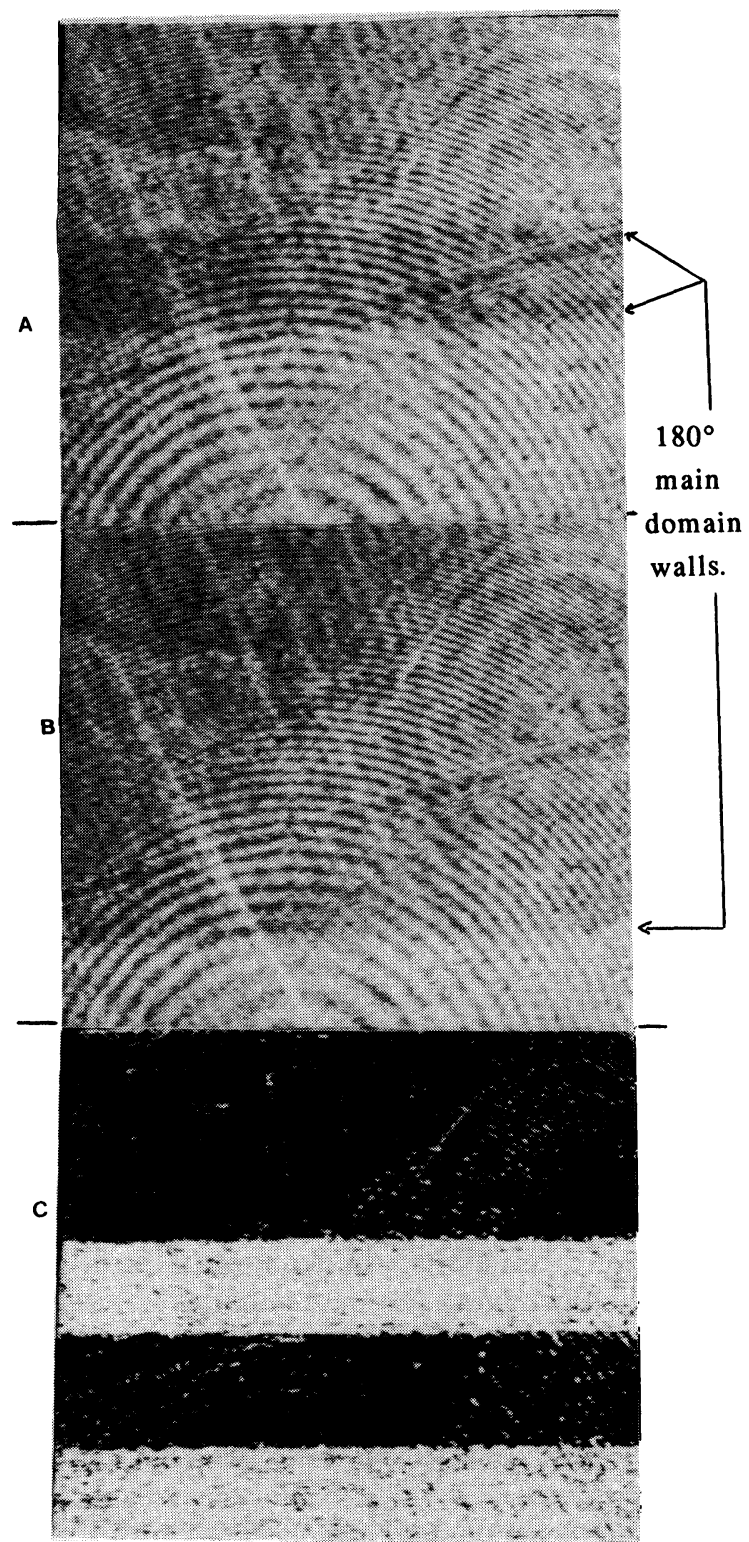

Figure 4 a: Reference image (Im 1), b: Image to substract $(\operatorname{Im} 2)$, c: Result image (Im 3). 
with bad visual quality; but, laser light (used for magnetic domain observation) speckles and surface specimen defects are attenuated. At the same time, the contrast is also enhanced by this processing.

The same series shows up the dispersion in motion of walls even if they limit the same magnetic domain as demonstrated by the non-identical width of the dark areas in the processed image. Similarly, it also happens that, under the same excitation conditions, some parts of the same wall show distinct motion one from the other.

According to the aforementioned experimental proof, the calculated wall displacement must result from different computing levels. At first, each wall pixel displacement is measured and an individual wall histogram for its 512 pixels is drawn, giving a mean value of pixel travelling distance for this wall. Secondly, similar processing is performed for all grain walls giving a more generalized histogram than the previous one and, consequently, an average wall displacement for this selected grain. The histogram displayed on Figure 5 illustrates the results obtained after these two computing levels, in the case of one grain of the specimen. The distribution allure is approximatively gaussian.

As shown in this example, the walls in the vicinity of grainboundaries move less rapidly than the others, probably because of the supplementary pinning effect due to the grainboundaries. This is specially the case for areas with uniformly distributed defects.

The previous algorithm was extended to some other specimen grains having distinct misorientations. These latter have been measured by $\mathrm{X}$-ray back reflection and controlled, additionally, by measurement of the magnetic domain widths in the demagnetized state.

Assuming the same number of walls undergoing magnetization process, the experimental curves obtained (Figure 6) show an average wall displacement in the laser treated specimen less than in the same specimen before irradiation.

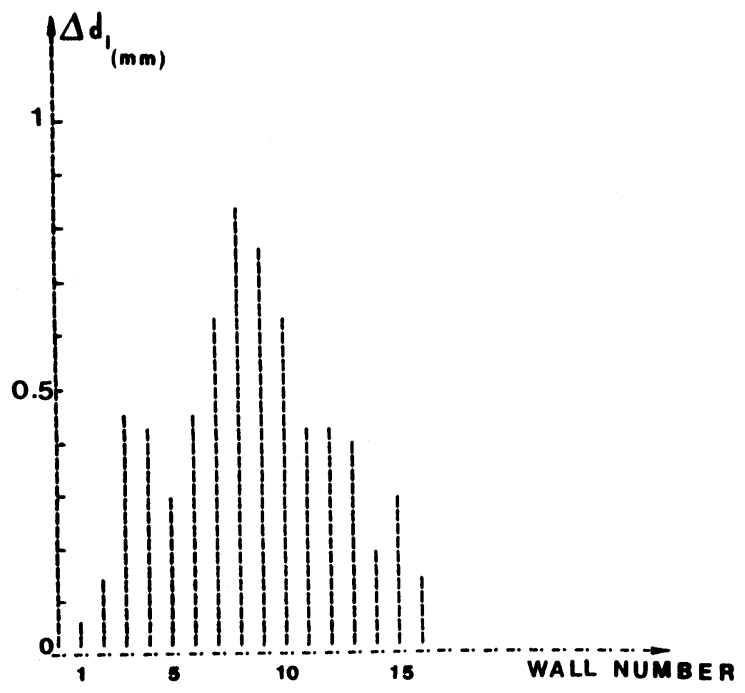

Figure 5 "Like-gaussian" mean displacement distribution for a series of walls in the same grain. 


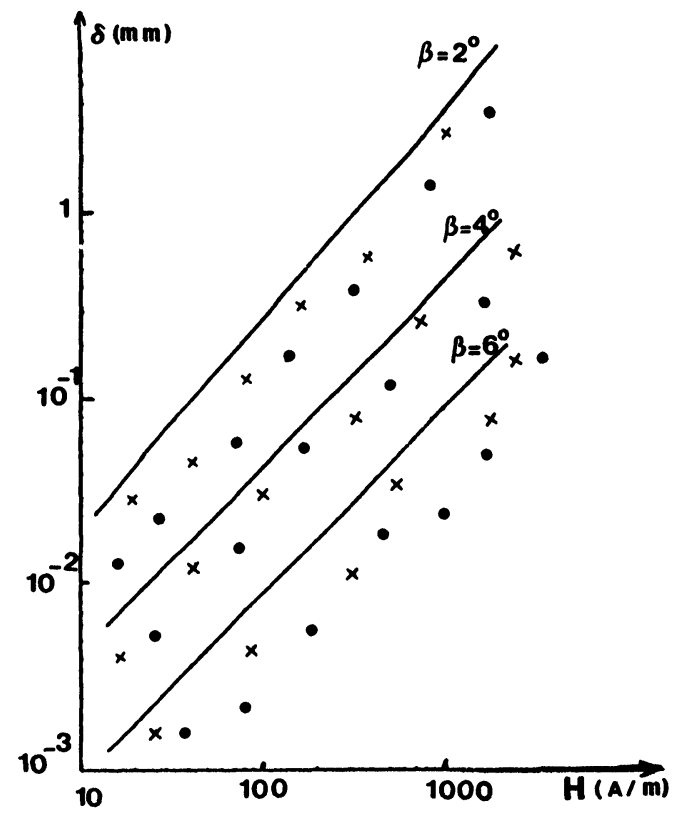

Figure 6 Experimental and theoretical mean wall displacement in single grains.

\section{THEORETICAL CONSIDERATIONS AND DISCUSSION}

The magnetic patterns considered here are the most current in the commercial $\mathrm{Si}-\mathrm{Fe}$ sheets: pure slab domains or slab domains associated with lancets. Therefore, assuming an unstressed sample, the total energy $E_{T}$ per unit area of a specimen surface, in the international MKS system of units, is given by the following equation:

$$
E_{T}=E_{P}+E_{W}
$$

Where $E_{P}$ is the magnetostatic energy due to the perpendicular component of magnetization in stripe domains and $E_{W}$ is the wall energy due to both main walls and walls of transverse $90^{\circ}$ domains connecting the two visible parts of a lancet on the specimen surface.

Therefore, the previous expression becomes:

$$
\begin{aligned}
E_{T}= & \frac{8 d}{1+\bar{\mu}^{*}} \frac{M_{s}^{2} \sin ^{2} \beta}{\mu_{0} \pi^{3}}\left(2 n S_{s 2}-1\right)^{2} \\
& +\gamma_{180}\left(\frac{t}{d \cos \alpha}+n S_{s 1}\right)+\gamma_{90} n S_{t}
\end{aligned}
$$

Where $M_{s}$ is the saturation magnetization, $d$ the main domain width in the demagnetized state, $n$ the density of lancets, $S_{s 1}$ and $S_{t}$ the surface areas respectively of the lancet part at the surface region of the specimen and of the transverse $90^{\circ}$ domains. $\gamma_{180}$ and $\gamma_{90}$ are wall energies of the $180^{\circ}$ and $90^{\circ}$ walls, 
respectively, $t$ is the specimen thickness, $\bar{\mu}^{*}$ the effective permeability of a domain and $\alpha$ the angle from the $180^{\circ}$ main wall to the normal to the specimen surface. $S_{s 2}$ is the area of the visible part of lancet emerging at the surface of the specimen.

Assuming reversible process conditions where the magnetic losses do not occur due to wall displacement and where the number of walls does not change during magnetization, those magnetic energies which vary by an application of fields are a magnetostatic energy $E_{P}$ and energy $E_{H}$ supplied by the field. In the range of $0<\delta / d<0.5$, the increase in the total energy $e_{T}$ with increasing in-plane excitation field in the rolling direction ${ }^{7}$ is:

$$
e_{T}=\frac{2 t}{\bar{\mu}^{*}} \frac{M_{s}^{2} \sin ^{2} \beta}{\mu_{0} d^{2}} \delta^{2}-\frac{2 M_{s}(\cos \beta) H t}{d} \delta
$$

The reduction of this quantity to a minimum with respect to the displacement $\delta$ of the $180^{\circ}$ domain wall gives the following expression:

$$
\delta=\frac{\mu_{0} \bar{\mu}^{*} d \cos \beta}{2 M_{s} \sin ^{2} \beta} H
$$

$\bar{\mu}^{*}$ is related to the static permeability $\bar{\mu}$ by the formula:

$$
\bar{\mu}^{*}=(\bar{\mu}-1) \frac{\sin ^{2} \beta}{\cos \beta}
$$

According to M. Imamurra et al.,$^{7}$ the calculated values of $\tilde{\mu}$ are 40000,10000 , 4000 and 2000 , at $\beta=2^{\circ}, 4^{\circ}, 6^{\circ}$ and $8^{\circ}$ respectively. So, the dependance of effective permeability on angle of inclination is deduced.

The $180^{\circ}$ main domain wall spacing in the demagnetized state, is given by the relation established by $\mathrm{C}$. Kittel: ${ }^{9}$

$$
d=\sqrt{\frac{\gamma_{180} t}{1.7 M_{s}^{2} \frac{2}{1+\mu^{*}}}} \frac{1}{\sin \beta}
$$

from which the ratio of $d_{1}$ for $\beta_{1}$ to $d_{2}$ for $\beta_{2}$ is expressed in terms of the ratio of $\sin \beta_{2}$ to $\sin \beta_{1}$ :

$$
\frac{d_{2}}{d_{1}}=\frac{\sin \beta_{1}}{\sin \beta_{2}}
$$

From the image observations, one couple of $d, \beta$ values was chosen as a reference point.

The previous data provides a set of straight lines (Figure 6) which over-estimate the real travel of the $180^{\circ}$ wall in our specimens. Besides, a correction must be made in order to take into account a threshold field required initially to move the wall from its equilibrium position in the demagnetized state. Thus in Eq. (4), $H$ becomes $\mathrm{H}-H_{p}$ where $H_{p}$ is the wall propagating field. According to experimental results, the value of $H_{p}$ is greater in irradiated samples than in non-irradiated ones. This may be attributed, partially, to the residual pinning effect caused by laser spots; but, the new subdomains nucleated along laser irradiation spot lines 
weaken this domain wall pinning due to free poles or internal stress resulting from the laser processing.

As is known, ${ }^{9,10}$ core losses are related both to the main domain wall spacing in the demagnetized state and to the domain wall velocity. Now, as the walls become more closely spaced, the eddy currents from neighbouring walls interact and the net result is an increase in the wall motion damping coefficient and a decrease in wall mobility. Some previous experimental results ${ }^{1}$ confirm our remark by the means of the relationship between total losses and average $180^{\circ}$ main domain wall spacing. So, in certain circumstances, core losses may be greater after laser treatment than before irradiation.

Practically, the optimization of laser spot spacing produces a satisfactory main domain width. Besides, more domain walls are active in the irradiated sheets because of the reverse subdomains induced by laser irradiation. Consequently, the behaviour of losses of irradiated specimens is conditioned by the reverse subdomains.

\section{CONCLUSIONS}

From this investigation of some commercial $\mathrm{Hi}-\mathrm{B}$ specimens, several conclusions are derived as follows:

1. A certain shape irregularity is observed in the main magnetic domains of irradiated sheets. This irregularity becomes more pronounced if the laser spot spacings are not optimized.

2. This sort of laser treatment causes some pinning effect which increases the threshold field needed to move the main domain wall initially.

3. The refining process induces an increase in spread of wall motion and a decrease in wall mobility.

4. In the irradiated samples, the iron losses are related to the reverse subdomains behavior.

\section{ACKNOWLEDGMENT}

The author wishes to thank Prof. G. Pierron, A. Mailfert and Mr G. Bean for their helpful discussions and encouragement.

\section{References}

1. Nozawa, T., Yamamoto, T., Matsuo, Y. and Ohya, Y. (1979). I.E.E.E. Trans. Mag MAG-15, pp 972-981.

2. Fukawa, K. and Yamamoto, T. (1982). I.E.E.E. Trans. Mag, MAG-18, pp 963-969.

3. Nakamura, M., Hirose, K., Nozawa, T. and Matsuo, M. (1987). I.E.E.E. Trans. Mag, MAG-23, pp 3074-3076.

4. Yabumoto, M., Kobayashi, H., Nozawa, T., Hirose, K. and Takahashi, N., EG-01 Intermag 87, Japan. 
5. Nozawa, T., Matsuo, Y., Kobayashi, H., Iwayama, K. and Takahashi, N. (1988). J. Appl. Phys. 63(8) pp 2966-2970.

6. Barradi, T., Mailfert, A., Mahieu, A. and Piot, P. Journées d'Electrotechnique 88, Paris Mars 1988.

7. Imamura, M., Sasaki, T. and Yamaguchi, T. (1984). I.E.E.E. Trans. Mag, MAG-20, pp 2120-2129.

8. Kittel, C. (1966). Rev. Mod. Phys, 21, pp 702-710.

9. Pry, R. H. and Bean, C. P. (1958). J. Appl. Phys, 29, pp 532-533.

10. Williams, H. J., Shockley, W. and Kittel, C. (1950). Phy. Rev, 80, pp 1090-1094. 